\title{
Adverse muscle composition predicts all-cause mortality in the UK Biobank imaging study
}

\author{
Jennifer Linge ${ }^{1,2 *}$ (D), Mikael Petersson ${ }^{1}$, Mikael F. Forsgren ${ }^{1,2,3}$, Arun J. Sanyal ${ }^{4}$ \& Olof Dahlqvist Leinhard ${ }^{1,2,3}$ \\ ${ }^{1}$ AMRA Medical, Linköping, Sweden; ${ }^{2}$ Department of Health, Medicine and Caring Sciences, Linköping University, Linköping, Sweden; ${ }^{3}$ Center for Medical Image Science and \\ Visualization (CMIV), Linköping University, Linköping, Sweden; ${ }^{4}$ Department of Internal Medicine and Division of Gastroenterology, Hepatology and Nutrition, Virginia \\ Commonwealth University, Richmond, VA, USA
}

\begin{abstract}
Background Adverse muscle composition (MC) as measured by magnetic resonance imaging has previously been linked to poor function, comorbidity, and increased hospitalization. The aim of this study was to investigate if adverse MC predicts all-cause mortality using data from UK Biobank.

Methods There were 40178 participants scanned using a 6 min magnetic resonance imaging protocol. Images were analysed for thigh fat-tissue free muscle volume and muscle fat infiltration (MFI) using AMRA ${ }^{\circ}$ Researcher (AMRA Medical, Linköping, Sweden). For each participant, a sex, weight, and height invariant muscle volume z-score was calculated. Participants were partitioned into four MC groups: (i) normal MC, (ii) only low muscle volume $[<25$ th percentile for muscle volume z-score (population wide)], (iii) only high MFI [ $>75$ th percentile (population wide, sex-specific)], and (iv) adverse MC (low muscle volume z-score and high MFI). Association of MC groups with mortality was investigated using Cox proportional-hazard modelling with normal MC as referent (unadjusted and adjusted for low hand grip strength, sex, age, body mass index, previous diagnosis of disease (cancer, type 2 diabetes and coronary heart disease), lifestyle, and socioeconomic factors (smoking, alcohol consumption, physical activity, and Townsend deprivation index).

Results Muscle composition measurements were complete for 39804 participants [52\% female, mean (SD) age 64.2 (7.6) years and body mass index $\left.26.4(4.4) \mathrm{kg} / \mathrm{m}^{2}\right]$. Three hundred twenty-eight deaths were recorded during a follow-up period of 2.9 (1.4) years after imaging. At imaging, adverse $M C$ was detected in $10.5 \%$ of participants. The risk of death from any cause in adverse MC compared with normal MC was 3.71 (95\% confidence interval 2.81-4.91, $P<0.001)$. Only low muscle volume and only high MFI were independently associated with all-cause mortality [1.58 (1.13-2.21), $P=0.007$, and 2.02 (1.51-2.71), $P<0.001$, respectively]. Adjustment of low hand grip strength [1.77 (1.28-2.44), $P<0.001]$ did not attenuate the associations with any of the MC groups. In the fully adjusted model, adverse MC and only high MFI remained significant $(P<0.001$ and $P=0.020)$ while the association with only low muscle volume was attenuated to non-significance $(P=0.560)$. The predictive performance of adverse MC $[1.96$ (1.42-2.71), $P<0.001$ ] was comparable with that of previous cancer diagnosis [1.93 (1.47-2.53), $P<0.001]$ and smoking [1.71 (1.02-2.84), $P=0.040]$. Low hand grip strength was borderline non-significant [1.34 (0.96-1.88), $P=0.090]$.

Conclusions Adverse $M C$ was a strong and independent predictor of all-cause mortality. Sarcopenia guidelines can be strengthened by including cut-offs for myosteatosis enabling detection of adverse MC.
\end{abstract}

Keywords Sarcopenia; Magnetic resonance imaging; Frailty; Myosteatosis; Muscle fat infiltration 


\section{Introduction}

Whether suffering in later or end stages of a disease, from metabolic disorders, or of natural effects from aging, maintaining muscle health and functional performance is of high importance. ${ }^{1-4}$ Older adults progressively lose muscle mass and strength as they age-a process that is intensified in individuals with metabolic disorders, like type 2 diabetes and non-alcoholic steatohepatitis, and is rapidly occurring in end-stage diseases like cancer, cirrhosis, or chronic kidney disease. ${ }^{5-10}$

Sarcopenia is a muscle disorder characterized by progressive and generalized loss of skeletal muscle mass, strength, and/or physical performance, and predisposes individuals to adverse health outcomes. ${ }^{3,4}$ Although consensus has not yet been reached on the definition of sarcopenia, it has moved from focusing only on muscle mass to include muscle function, and today, there is growing evidence of the importance of myosteatosis as a measure of muscle quality. ${ }^{11-14}$

Studies investigating the associations between muscle mass or sarcopenia and mortality show ambiguous results. ${ }^{15-17}$ Some studies indicate that muscle strength, not mass, is more important to assess. ${ }^{4,15}$ Others suggest that muscle mass is predictive of adverse outcomes and mortality; however, they note that previous techniques used to assess muscle mass, and/or commonly used body size normalizations, have obscured this relationship. ${ }^{13,17-19}$ Throughout the literature, a wide variety of techniques have been used to measure muscle mass [bioelectrical impedance (BIA), dual-energy X-ray absorptiometry (DXA), deuterated creatine $\left(\mathrm{D}_{3} \mathrm{Cr}\right)$ dilution, single slice computer tomography $(\mathrm{CT})$, or magnetic resonance imaging (MRI)] and sarcopenia has been identified in many different ways (e.g. by using cut-offs for muscle mass alone or together with a functional measure like hand grip strength, walking pace or stair climbing).,4,17 In addition, different health outcomes have been used to validate the diagnostic criteria and/or technique investigated. Still, the precise role of muscle mass in relation to functional performance, adverse outcomes, and mortality remains unsolved.

Studies investigating the associations between myosteatosis (also called intramuscular fat or muscle fat infiltration) and mortality are generally consistent in showing a strong link to mortality. ${ }^{20-22}$ However, as myosteatosis cannot be assessed using simpler techniques such as BIA or DXA, the vast majority of data seen in current literature is from populations where CT is routinely used. Studies utilizing CT data commonly rely on opportunistic assessments of skeletal muscle attenuation as a surrogate for muscular fat deposition from single slice assessments. Further, they are restricted to specific patient groups like cancer populations, critically ill patients or patients with cirrhosis. ${ }^{11,21-23}$ There are also studies of such populations showing the predictive performance of the combined assessment of muscle mass and myosteatosis using opportunistic CT. ${ }^{22,24}$ However, few (if any) studies have investigated the association of the collective description of muscle mass and myosteatosis with adverse outcomes or mortality in a general aging population.

In recent years, methods quantifying muscle composition with high accuracy and precision have been developed using 3D-volumetric MRI coupled with advanced image processing techniques. ${ }^{25-28} \mathrm{~A}$ rapid and standardized MRI protocol enabling muscle composition assessment is currently running in the large UK Biobank imaging study, ${ }^{29}$ where more than 40000 participants have been imaged to date. Based on this large population study, the collective description of MRI-measured fat-tissue free muscle volume (FFMV) and muscle fat infiltration (MFI) has been shown to increase the predictive performance of increased hospitalization and poor function, and recently, cut-points to identify an adverse muscle composition phenotype have been published. ${ }^{13,14,19}$ These cut-points have been tested in a non-alcoholic fatty liver disease (NAFLD) population of 1200 individuals. $^{14}$ The condition was prevalent in $14 \%$ of those with NAFLD and was associated with 1.7-2.4 times higher prevalence of poor function, as well as 2.1 times, and 3.3 times higher prevalence of type 2 diabetes and coronary heart disease. ${ }^{14,19}$

The current evidence indicates that assessment of adverse muscle composition identifies vulnerable individuals both within the general population and metabolic diseases. ${ }^{13,14,19}$ Prospective studies linking adverse muscle composition to adverse outcomes are needed to determine whether identification of this condition could guide treatment strategies or motivate preventive healthcare. The aim of this work was to investigate if adverse muscle composition predicts all-cause mortality in the UK Biobank imaging study.

\section{Materials and methods}

This study was based on the 40178 participants first scanned in the UK Biobank imaging study. UK Biobank is a long-term study following 500000 volunteers 40-69 years of age at recruitment in 2006-2010. ${ }^{29}$ As a sub-study, 100000 participants are being recalled for a detailed imaging assessment, including repeat of baseline assessment.

This research was conducted using the UK Biobank resource, project ID 6569. The study was approved by the North West Multicenter Research Ethics Committee, UK. Written informed consent was obtained prior to study entry.

\section{Inclusion}

Participants were required to have known sex, age, weight, height, and complete description of muscle composition (FFMV and MFI of at least one leg). 


\section{Muscle composition measurements}

The participants were scanned in a Siemens MAGNETOM Aera 1.5-T MRI scanner (Siemens Healthineers, Erlangen, Germany) using a $6 \mathrm{~min}$ dual-echo Dixon Vibe protocol, providing a water and fat separated volumetric data set covering neck to knees. Body composition analyses were performed using AMRA $^{\circledR}$ Researcher (AMRA Medical AB, Linköping, Sweden). ${ }^{25-28}$ The supporting information provides details of in vivo acquisitions and analysis. Muscle composition measurements and the definition of adverse $M C$ has been previously described and utilized in other studies. ${ }^{13,14,19}$ The MRI measurements utilized in this study are publicly available through UK Biobank (Category 149, Abdominal composition):

- Fat-tissue free muscle volume (FFMV, referred to as 'muscle volume'): FFMV is defined as the volume of all voxels with fat fraction $<50 \%$ ('viable muscle tissue') in the thighs. This is to be differentiated from lean muscle volume (sometimes called 'contractile muscle volume') calculated by subtracting the fat volume from the entire muscle volume. ${ }^{26,27}$ If data were missing for one leg, the total thigh muscle volume was estimated through multiplication by two of the other leg.

- Fat-tissue free muscle volume z-score (referred to as 'muscle volume z-score'): For each participant, a matched virtual control group (VCG) was stratified among the study participants with complete muscle composition data. Each participant's VCG included at least 150 individuals with the same sex and with similar body mass index (BMI). Based on each VCG, a personalized muscle volume z-score was calculated measuring how many standard deviations each participant was from the mean thigh FFMV/height ${ }^{2}$ of their VCG. ${ }^{13}$ This variable is sex, weight, and height invariant and has been associated with poor function and increased hospitalization. ${ }^{13}$

- Muscle fat infiltration (MFI): MFI is defined as the mean fat fraction in the 'viable muscle tissue' (FFMV) of the right and left anterior thighs. ${ }^{26,27}$ If data were missing for one leg, the mean MFI was estimated by the MFI of the other leg. Due to the difference in magnitude between female participants (higher) and male participants (lower), a sex-adjusted MFI was included in tables calculated by subtracting the sex-specific population median for each participant.

- Adverse muscle composition (MC): Adverse $M C$ is defined by low muscle volume coupled with high $\mathrm{MFI}^{14}$ As the muscle volume z-score is sex invariant, ${ }^{13}$ 'Low muscle volume' was defined as $<25$ th percentile of the whole cohort (participants included in this study) for both female and male participants (muscle volume z-score $<-0.68 \mathrm{SD}$ ). As the magnitude of MFI differs between female and male participants, ${ }^{13,14,25}$ 'high $\mathrm{MFI}^{\prime}$ was defined as $>75$ th percentile for female and male participants separately [MFI > 8.82\% (female participants), 7.69\% (male participants)]. Figure S1 provides a scatter plot of muscle volume z-score and MFI including sex-specific distributions of the variables and illustration of the cut-points for adverse $M C$.

\section{Data collection UK Biobank}

Mortality data were obtained through UK Biobank's linkage to national death registries. Height was recorded using a Seca stadiometer (Seca GMBH, Hamburg, Germany), and weight with a Tanita BC418ma (Tanita Europe, Amsterdam, The Netherlands). Previous diagnoses of cancer, coronary heart disease and type 2 diabetes were based on electronic health care records (accessed November 2020, available from April 1992 to August 2020) in combination with self-reported information collected via interviews with trained nurses. Information on smoking, alcohol consumption, and physical activity were acquired through touchscreen questionnaires, and the Townsend deprivation index was calculated by UK Biobank immediately prior to each participant joining UK Biobank. Further details on variable definitions can be found in the supporting information.

Measures of functional performance and frailty

- Hand grip strength was measured using a Jamar J00105 hydraulic hand dynamometer (Lafayette Instrument, Lafayette, IN, USA) (protocol in supporting information). Handedness was collected through the touchscreen questionnaires, and the data recorded for the dominant hand were used. If information of handedness was missing or a participant reported using both hands, the mean of the right and left hand was used. Low hand grip strength was defined using the sex-specific cut-offs recommended by the European Working Group on Sarcopenia in Older People (EWGSOP2) (16/27 kg for female participants/male participants). ${ }^{3}$

- Walking pace was collected through the touchscreen questionnaires with categories 'slow pace', 'steady average pace', and 'brisk pace' (touchscreen screenshot in supporting information).

- Falls was collected through the touchscreen questionnaires with categories 'no falls', 'only one fall', 'more than one fall' in the last year (touchscreen screenshot in supporting information).

\section{Statistical analysis}

Associations between muscle composition variables and all-cause mortality

The association between muscle volume z-score and $\mathrm{MFI}$ with all-cause mortality was investigated using Cox proportional-hazard models with days after the time of 
imaging as timescale. An unadjusted model including muscle volume z-score (continuous) and MFI (continuous) (M0) was implemented followed by models subsequently adjusted for hand grip strength (continuous) (M1), sex, age and BMI $(\mathrm{M} 2)$, previous disease (diagnosis of cancer, coronary heart, and disease type 2 diabetes prior to imaging) (M3), and lifestyle and socioeconomic factors (alcohol consumption, smoking status, physical activity level, and Townsend deprivation index) (M4). The interaction effect of muscle volume z-score and $\mathrm{MFI}$ on all-cause mortality was tested by including the corresponding interaction term in model $\mathrm{MO}$.

Associations between adverse muscle composition and all-cause mortality

The participants were categorized into one out of four muscle composition groups using the cut-points for adverse MC: (i) normal MC, (ii) only low muscle volume (low muscle volume z-score), (iii) only high MFI, and (iv) adverse MC. The associations of muscle composition with all-cause mortality were investigated using Kaplan-Meier survival curves and Cox proportional-hazard models with days after the time of imaging as timescale. Adjustments were subsequently added according to models M1-M4 described earlier but with a binary representation of hand grip strength (cut-offs 16/27 kg for female participants/male participants ${ }^{3}$ ).

Combining adverse muscle composition with measures of functional performance and frailty

Three combined variables were created using adverse $M C$ (yes/no), hand grip strength (high/low), slow walking pace (no/yes) and $>1$ fall last year (no/yes), and associated with all-cause mortality using unadjusted Cox proportional-hazard models with days after imaging as timescale and adverse $M C=$ no, hand grip strength = high; adverse $M C=$ no, slow walking pace $=$ no; adverse $M C=$ no, and $>1$ fall last year $=$ no as referents.

An experienced statistician independently replicated the results. Computations were performed using $\mathrm{R}$ version 3.4.4 (The R Foundation, Vienna, Austria). Associations were considered significant for $\alpha<0.05$. The supporting information lists $R$ packages used for analysis and visualizations.

\section{Patient and public involvement}

It was not appropriate or possible to involve patients or the public in the design, or conduct, or reporting, or dissemination plans of our research.

\section{Results}

Of the 40178 participants, 39804 (99\%) had complete data for sex, age, height, weight, and muscle composition and were included for analysis. The mean age was 64.2 years (SD 7.6), 52\% were female, and the mean BMI was 26.4 (SD 4.4) kg/m². $. N=24061$ (61\%) of 39804 participants presented with normal MC, N = 5791 (15\%) with only low muscle volume, $N=5785$ (15\%) with only high MFI and $N=4167$ (11\%) with adverse MC (Table 1). Tables 2 and 3 present sex-specific data by muscle composition groups. The distribution of muscle composition was similar between female and male participants. For both female and male participants, the highest prevalence of disease (cancer, coronary heart disease, and type 2 diabetes) was found within the adverse $M C$ group. The highest BMI was found in the only high MFI group and the oldest participants in the adverse $M C$ group.

During a mean follow-up of 2.9 (SD 1.4) years and 114862 person-years at risk, 328 (out of 39 804) individuals died (209 male and 119 female participants). Figure 1 shows the breakdown of primary and secondary causes of death across ICD-10 blocks and chapters. The most common primary and secondary causes of death were recorded within Chapter II (neoplasms, 37.2\% of ICD-10 codes) and Chapter IX (diseases of the circulatory system, $29.1 \%$ of ICD-10 codes). Table 4 show the most common primary and secondary causes of death per ICD-10 code block and the distribution of count and incidence across the different MC groups. The most common causes of death were cancers of the digestive system ( $N=88$ codes) and ischaemic heart disease ( $N=74$ codes). Although numbers for specific causes of death are still low, the incidence of death was generally higher in adverse $M C$ than compared with the other three muscle phenotypes. For some ICD-10 blocks, the only high MFI group showed as high (or even higher) mortality as adverse MC. This was true for 130-I52 (other forms of heart disease), I10-I15 (hypertensive disease), C81-C96 (malignant neoplasms, stated or presumed to be primary, of lymphoid, haematopoietic and related tissue), I26-I28 (pulmonary heart disease and diseases of pulmonary circulation), and Covid-19. Figure S2 provides a visual representation of Table 4.

\section{Associations between muscle composition variables and all-cause mortality}

Cox proportional-hazards modelling the continuous muscle composition variables showed that muscle volume z-score and MFI were both significantly, and independently, associated with all-cause mortality [hazard ratios (HRs): 0.70 (95\% confidence interval 0.62-0.79), $P<0.001$, and 1.10 (1.05-1.15), $P<0.001]$. The interaction between muscle volume z-score and MFI was not significant $(P=0.116)$. In the model including hand grip strength, muscle volume z-score and MFI remained significant [0.70 (0.62-0.79), $P<0.001$; 1.12 (1.06-1.17), $P<0.001$ ] while hand grip strength showed non-significance [1.01 (1.00-1.02), $P=0.053]$. In the fully adjusted model, MFI remained significant [1.09 (1.01-1.16), 
Table 1 Population characteristics

\begin{tabular}{|c|c|c|c|}
\hline & Total & Female participants & Male participants \\
\hline$N$ & 39804 (100.0\%) & $20718(52.1 \%)$ & $19,086(47.9 \%)$ \\
\hline Sex (female/male) & $52.1 \% / 47.9 \%$ & $100.0 \% / 0.0 \%$ & $0.0 \% / 100.0 \%$ \\
\hline Age (years) & $64.20(7.55)$ & $63.54(7.40)$ & $64.92(7.64)$ \\
\hline BMI $\left(\mathrm{kg} / \mathrm{m}^{2}\right)$ & $26.41(4.37)$ & $25.97(4.72)$ & 26.89 (3.89) \\
\hline Waist circumference (cm) & $88.33(12.66)$ & $82.83(11.84)$ & $94.28(10.69)$ \\
\hline FFMV, total thigh (L) & $10.17(2.55)$ & $8.19(1.17)$ & $12.33(1.76)$ \\
\hline FFMV, left anterior thigh (L) & $1.70(0.48)$ & $1.34(0.23)$ & $2.10(0.36)$ \\
\hline FFMV, right anterior thigh (L) & $1.71(0.49)$ & $1.34(0.23)$ & $2.11(0.36)$ \\
\hline FFMV, left posterior thigh (L) & $3.35(0.80)$ & $2.74(0.38)$ & $4.02(0.57)$ \\
\hline FFMV, right posterior thigh (L) & $3.40(0.82)$ & $2.76(0.39)$ & $4.08(0.57)$ \\
\hline FFMV, z-score (SD) & $-0.01(0.98)$ & $-0.01(0.99)$ & $-0.01(0.98)$ \\
\hline MFI, mean anterior thigh (\%) & $7.34(1.88)$ & $7.82(1.87)$ & $6.81(1.75)$ \\
\hline MFI, left anterior thigh (\%) & $7.50(1.91)$ & $8.01(1.90)$ & $6.93(1.75)$ \\
\hline $\mathrm{MFI}$, right anterior thigh (\%) & $7.19(1.92)$ & $7.65(1.91)$ & $6.70(1.81)$ \\
\hline MFI, left posterior thigh (\%) & $11.06(2.41)$ & $11.62(2.34)$ & $10.44(2.33)$ \\
\hline MFI, right posterior thigh (\%) & $10.85(2.42)$ & $11.43(2.34)$ & $10.22(2.34)$ \\
\hline MFI, sex-adjusted (pp) & $0.27(1.81)$ & $0.27(1.87)$ & $0.28(1.75)$ \\
\hline Cancer (yes/no/missing) & $10.4 \% / 88.7 \% / 0.9 \%$ & $10.9 \% / 88.1 \% / 1.0 \%$ & $9.8 \% / 89.3 \% / 0.8 \%$ \\
\hline Type 2 diabetes (yes/no/missing) & $5.0 \% / 94.5 \% / 0.5 \%$ & $3.2 \% / 96.2 \% / 0.6 \%$ & $7.0 \% / 92.6 \% / 0.4 \%$ \\
\hline Coronary heart disease (yes/no/missing) & $5.2 \% / 94.4 \% / 0.4 \%$ & $2.6 \% / 96.9 \% / 0.4 \%$ & $7.9 \% / 91.7 \% / 0.4 \%$ \\
\hline Hand grip strength $=$ low (yes/no/missing) & $7.1 \% / 89.7 \% / 3.2 \%$ & $7.1 \% / 89.5 \% / 3.4 \%$ & $7.1 \% / 89.9 \% / 3.0 \%$ \\
\hline Smoking (no/previous/current/missing) & $61.9 \% / 33.6 \% / 3.5 \% / 1.0 \%$ & $65.5 \% / 30.5 \% / 2.9 \% / 1.1 \%$ & $58.1 \% / 37.0 \% / 4.1 \% / 0.9 \%$ \\
\hline Alcohol consumption (g/day) & $22.77(25.47)$ & $16.67(19.66)$ & $29.39(29.14)$ \\
\hline $\begin{array}{l}\text { Physical activity IPAQ } \\
\text { (moderate/low/high/missing) }\end{array}$ & $43.6 \% / 19.7 \% / 36.0 \% / 0.8 \%$ & $44.2 \% / 21.5 \% / 33.4 \% / 0.8 \%$ & $42.9 \% / 17.6 \% / 38.8 \% / 0.7 \%$ \\
\hline Townsend deprivation index & $-1.91(2.71)$ & $-1.86(2.72)$ & $-1.95(2.71)$ \\
\hline
\end{tabular}

BMI, body mass index; FFMV, fat-tissue free muscle volume; IPAQ, international physical activity questionnaire; MFI, muscle fat infiltration. Low muscle volume defined as $<25$ th percentile of the whole cohort for muscle volume z-score [cut-point -0.68 (both female participants and male participants)], and high muscle fat infiltration (MFI) as $>75$ th percentile of the whole cohort [cut-points $8.82 \%$ and $7.69 \%$ (female participants/male participants)]. Adverse MC defined as low muscle volume coupled with high MFI. ${ }^{8}$ Values are mean (standard deviation) for continuous variables and fraction per category for categorical and binary variables.

$P=0.020]$, while muscle volume $z$-score reached borderline significance $[0.87(0.76-1.01), P=0.064]$ and the association with hand grip strength was non-significant [0.99 (0.97-1.00), $P=0.162]$. Tables S1-S5 shows complete modelling results.

Associations between adverse muscle composition and allcause mortality Figure 2 shows the Kaplan-Meier survival curves for all-cause mortality by muscle composition phenotype. In the unadjusted Cox proportional-hazards model, the risk of death from any cause among those with adverse $M C$ compared with normal MC was 3.71 (95\% confidence interval 2.81-4.91), $P<0.001$. Only low muscle volume and only high $\mathrm{MFI}$ were also significantly associated with a higher risk for all-cause mortality as compared with normal muscle composition [1.58 (1.13-2.21), $P=0.007$, and 2.02 (1.51-2.71), $P<0.001$, respectively]. In the model including muscle composition groups and low hand grip strength, adverse $M C$, only low muscle volume, and only high $\mathrm{MFI}$ remained significant [3.39 (2.54-4.53), $P<0.001 ; 1.49$ (1.06-2.10), $P=0.020$; and 2.02 (1.51-2.70), $P<0.001$ ] and low hand grip strength was independently associated with increased mortality [1.77 (1.28-2.44), $P<0.001$ ]. For the fully adjusted model (including adjustment for low hand grip strength, sex, age, BMI, previous disease, lifestyle, and socioeconomic factors) (Figure 3), 37681 participants had complete data, among which 306 deaths were recorded. Results showed that adverse MC and only high MFI remained significantly associated with higher rates of mortality [1.96 (1.42-2.71), $P<0.001$, and 1.49 (1.08-2.07), $P=0.020$, respectively]. Other factors that reached level of significance in the fully adjusted model were sex [male 1.60 (1.25-2.05), $P<0.001)$, age (1.09 (1.07-1.11), $P<0.001]$, previous cancer diagnosis [1.93 (1.47-2.53), $P<0.001$ ], previous type 2 diabetes diagnosis [1.58 (1.092.29), $P=0.020]$, previous coronary heart disease diagnosis [1.46 (1.03-2.07), $P=0.040$ ], smoking status [current 1.71 (1.02-2.84), $P=0.040$ ] and physical activity [low (1.37 (1.03-1.82), $P=0.030]$. Low hand grip strength was non-significant [1.34 (0.96-1.88), $P=0.090]$. Tables S6-S10 show the complete modelling results.

\section{Combining adverse muscle composition with measures of functional performance and frailty}

Figure 4 shows the results from the combined assessment of adverse $M C$ with measures of functional performance and frailty (hand grip strength, walking pace, and falls). Results showed that within those with good function (high hand grip strength, average/brisk walking pace, or no falls last year), adverse MC was significantly associated with all-cause mortality (HRs 2.5-2.9, all $P<0.001$ ). In addition, among those with poor function (low hand grip strength, slow walking pace, or $>1$ fall last year), adverse MC was also significantly 


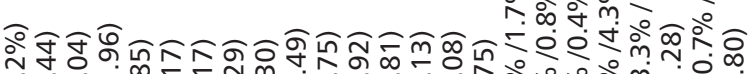

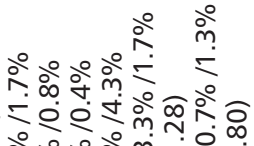
o 西

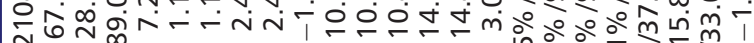
芒芒芒芒。

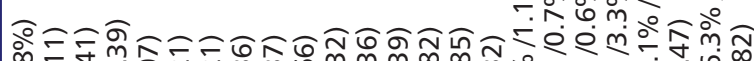

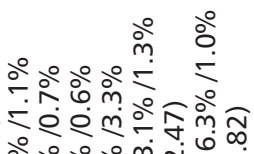

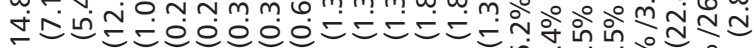

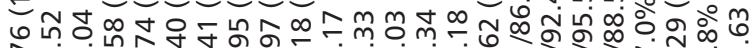
ठำ

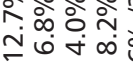
○े ๖े

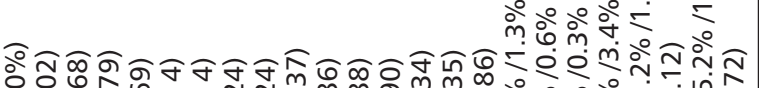

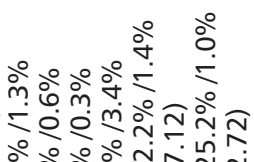

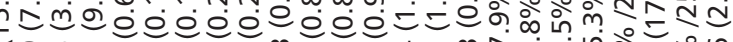

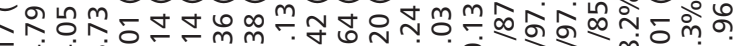

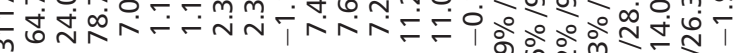
mont

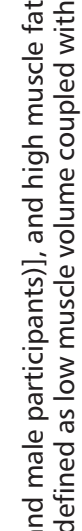
든

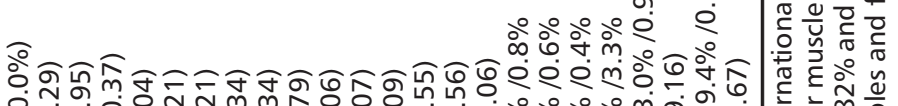
ใ् ๙

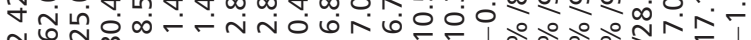




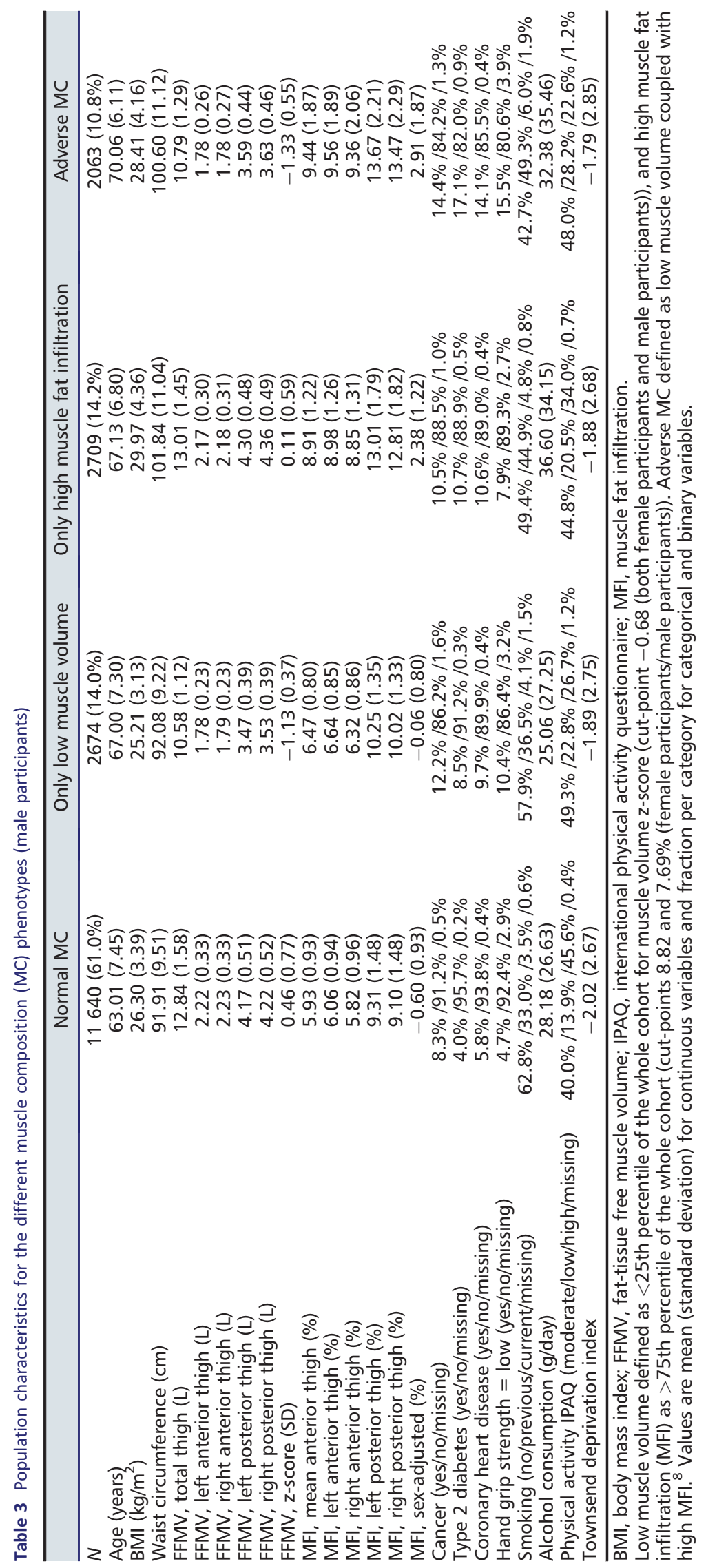




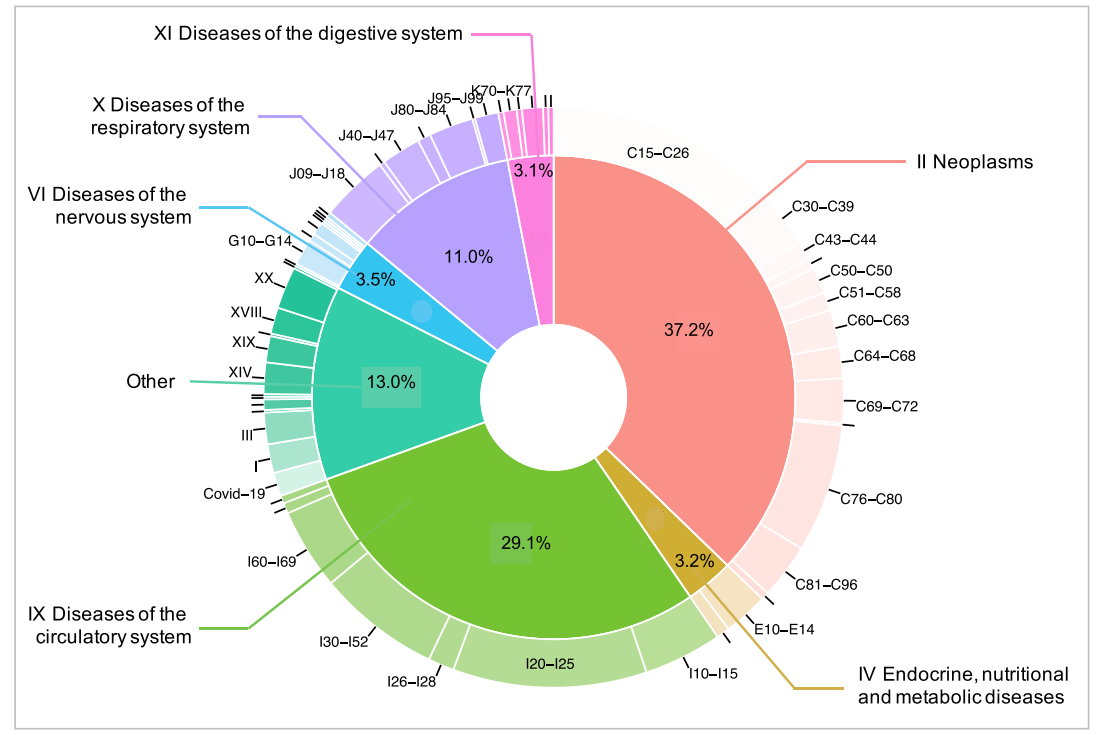

Figure 1 Breakdown of primary and secondary causes of death across ICD-10 blocks and chapters.

Table 4 Most common primary and secondary causes of death with distribution of codes and incidence within the different muscle composition (MC) groups

\begin{tabular}{|c|c|c|c|c|c|c|c|}
\hline ICD-10 block & $N$ & $\begin{array}{l}\text { Normal } \\
\text { MC }\end{array}$ & $\begin{array}{l}\text { Only low } \\
\text { muscle } \\
\text { volume }\end{array}$ & $\begin{array}{l}\text { Only high } \\
\text { muscle fat } \\
\text { infiltration }\end{array}$ & $\begin{array}{l}\text { Adverse } \\
\text { MC }\end{array}$ & $P$ value & Description \\
\hline C15-C26 & 88 & $38(1.58 \%$ o $)$ & $11(1.90 \%$ oo $)$ & 19 (3.28\%о) & $20(4.80 \%)$ & $<0.001$ & $\begin{array}{l}\text { Malignant neoplasms of digestive } \\
\text { organs }\end{array}$ \\
\hline $120-125$ & 74 & $29(1.21 \%$ o $)$ & $6(1.04 \%$ oo) & $17(2.94 \%$ o $)$ & $22(5.28 \%$ o $)$ & $<0.001$ & Ischaemic heart diseases \\
\hline C76-C80 & 49 & $19(0.79 \%$ o $)$ & $7(1.21 \%$ oo) & $6(1.04 \%)$ & $17(4.08 \%$ o $)$ & $<0.001$ & $\begin{array}{l}\text { Malignant neoplasms of ill- } \\
\text { defined, secondary and } \\
\text { unspecified sites }\end{array}$ \\
\hline $130-152$ & 47 & $15(0.62 \%$ & $3(0.52 \%$ o $)$ & 17 (2.94\%o) & $12(2.88 \%$ o $)$ & $<0.001$ & Other forms of heart disease \\
\hline $160-169$ & 31 & 17 (0.71\%о) & $4(0.69 \%)$ & $4(0.69 \%)$ & $6(1.44 \%$ o) & 0.249 & Cerebrovascular diseases \\
\hline I10-I15 & 30 & $7(0.29 \%$ oo) & $4(0.69 \%$ o $)$ & $12(2.07 \%$ o $)$ & $7(1.68 \%$ \%) & $<0.001$ & Hypertensive diseases \\
\hline J09-J18 & 26 & $10(0.42 \%)$ & $5(0.86 \%$ o $)$ & $4(0.69 \%$ o $)$ & $7(1.68 \%$ o $)$ & 0.007 & Influenza and pneumonia \\
\hline C30-C39 & 22 & $9(0.37 \%$ o $)$ & $1(0.17 \%$ о $)$ & $5(0.86 \%)$ & $7(1.68 \%)$ & 0.002 & $\begin{array}{l}\text { Malignant neoplasms of } \\
\text { respiratory and intrathoracic } \\
\text { organs }\end{array}$ \\
\hline C81-C96 & 21 & $9(0.37 \%$ o $)$ & $2(0.35 \%$ oo) & $8(1.38 \%$ oo $)$ & $2(0.48 \%$ o $)$ & 0.087 & $\begin{array}{l}\text { Malignant neoplasms, stated or } \\
\text { presumed to be primary, of } \\
\text { lymphoid, haematopoietic, and } \\
\text { related tissue }\end{array}$ \\
\hline C69-C72 & 17 & $8(0.33 \%$ o $)$ & $5(0.86 \%$ oo) & $2(0.35 \%$ oo & $2(0.48 \%$ oo $)$ & 0.604 & $\begin{array}{l}\text { Malignant neoplasms of eye, } \\
\text { brain, and other parts of central } \\
\text { nervous system }\end{array}$ \\
\hline E10-E14 & 17 & $2(0.08 \%$ o $)$ & $2(0.35 \%$ o $)$ & $5(0.86 \%$ o $)$ & $8(1.92 \%)$ & $<0.001$ & Diabetes mellitus \\
\hline J80-J84 & 17 & $5(0.21 \%)$ & $2(0.35 \%)$ & $5(0.86 \%)$ & $5(1.2 \% \circ)$ & 0.001 & $\begin{array}{l}\text { Other respiratory diseases } \\
\text { principally affecting the } \\
\text { interstitium }\end{array}$ \\
\hline $\mathrm{J} 40-\mathrm{J} 47$ & 15 & $4(0.17 \%$ oo $)$ & $0(0.00 \%)$ & $2(0.35 \%)$ & $9(2.16 \%)$ & $<0.001$ & Chronic lower respiratory diseases \\
\hline C60-C63 & 14 & $2(0.08 \%$ o $)$ & $3(0.52 \%)$ & $4(0.69 \%$ o $)$ & $5(1.20 \%)$ & $<0.001$ & $\begin{array}{l}\text { Malignant neoplasms of male } \\
\text { genital organs }\end{array}$ \\
\hline C64-C68 & 12 & $4(0.17 \%$ o $)$ & $3(0.52 \%)$ & $0(0.00 \%$ oo $)$ & $5(1.20 \%)$ & 0.014 & $\begin{array}{l}\text { Malignant neoplasms of urinary } \\
\text { tract }\end{array}$ \\
\hline C50-C50 & 10 & $6(0.25 \%)$ & $3(0.52 \%)$ & $1(0.17 \%$ oo) & $0(0.00 \%$ o $)$ & 0.452 & Malignant neoplasm of breast \\
\hline G10-G14 & 10 & $3(0.12 \%)$ & $1(0.17 \%$ oo $)$ & $3(0.52 \%)$ & $3(0.72 \%)$ & 0.011 & $\begin{array}{l}\text { Systemic atrophies primarily } \\
\text { affecting the central nervous } \\
\text { system }\end{array}$ \\
\hline
\end{tabular}

(Continues) 
Table 4 (continued)

\begin{tabular}{|c|c|c|c|c|c|c|c|}
\hline ICD-10 block & $N$ & $\begin{array}{l}\text { Normal } \\
\text { MC }\end{array}$ & $\begin{array}{c}\text { Only low } \\
\text { muscle } \\
\text { volume }\end{array}$ & $\begin{array}{l}\text { Only high } \\
\text { muscle fat } \\
\text { infiltration }\end{array}$ & $\begin{array}{l}\text { Adverse } \\
\text { MC }\end{array}$ & $P$ value & Description \\
\hline $126-128$ & 10 & $2(0.08 \%)$ & $1(0.17 \% \circ)$ & $4(0.69 \%)$ & $3(0.72 \%)$ & 0.002 & $\begin{array}{l}\text { Pulmonary heart disease and } \\
\text { diseases of pulmonary circulation }\end{array}$ \\
\hline Covid-19 & 9 & 1 (0.04\%o) & 1 (0.17\%о) & $4(0.69 \%$ o $)$ & $3(0.72 \% \circ)$ & $<0.001$ & Covid-19 \\
\hline J95-J99 & 9 & $3(0.12 \% \circ)$ & $0(0.00 \%)$ & $1(0.17 \%$ oo & $5(1.20 \%)$ & 0.001 & $\begin{array}{l}\text { Other diseases of the respiratory } \\
\text { system }\end{array}$ \\
\hline N17-N19 & 9 & $1(0.04 \%$ o $)$ & $2(0.35 \%)$ & $2(0.35 \% \circ)$ & $4(0.96 \%$ o $)$ & $<0.001$ & Renal failure \\
\hline K70-K77 & 8 & $2(0.08 \% \circ)$ & $0(0.00 \%)$ & $0(0.00 \%$ o $)$ & $6(1.44 \%$ o $)$ & $<0.001$ & Diseases of liver \\
\hline A30-A49 & 7 & $2(0.08 \%$ o & $1(0.17 \% \circ)$ & $2(0.35 \%)$ & $2(0.48 \% \circ)$ & 0.039 & Other bacterial diseases \\
\hline C43-C44 & 7 & $4(0.17 \% \circ)$ & $0(0.00 \%)$ & $0(0.00 \%$ o $)$ & $3(0.72 \%)$ & 0.178 & $\begin{array}{l}\text { Melanoma and other malignant } \\
\text { neoplasms of skin }\end{array}$ \\
\hline C51-C58 & 7 & $3(0.12 \% \circ)$ & $3(0.52 \% \circ)$ & $1(0.17 \%$ oo $)$ & $0(0.00 \%)$ & 0.928 & $\begin{array}{l}\text { Malignant neoplasms of female } \\
\text { genital organs }\end{array}$ \\
\hline R50-R69 & 7 & $5(0.21 \% \circ)$ & $0(0.00 \%)$ & $1(0.17 \%$ o) & $1(0.24 \% \circ)$ & 0.928 & General symptoms and signs \\
\hline Y83-Y84 & 6 & $1(0.04 \%$ o $)$ & $1(0.17 \%$ o & $2(0.35 \%)$ & $2(0.48 \%$ o) & 0.012 & $\begin{array}{l}\text { Surgical and other medical } \\
\text { procedures as the cause of } \\
\text { abnormal reaction of the patient, } \\
\text { or of later complication, without } \\
\text { mention of misadventure at the } \\
\text { time of the procedure }\end{array}$ \\
\hline D80-D89 & 5 & $2(0.08 \% \circ)$ & $0(0.00 \%$ o $)$ & $2(0.35 \%$ oo & $1(0.24 \%$ o $)$ & 0.167 & $\begin{array}{l}\text { Certain disorders involving the } \\
\text { immune mechanism }\end{array}$ \\
\hline E70-E90 & 5 & $1(0.04 \% \circ)$ & $0(0.00 \%$ o $)$ & $2(0.35 \% \circ)$ & $2(0.48 \%$ o $)$ & 0.008 & Metabolic disorders \\
\hline G30-G32 & 5 & $4(0.17 \%$ o $)$ & $1(0.17 \%$ o $)$ & $0(0.00 \%)$ & $0(0.00 \%)$ & 0.242 & $\begin{array}{l}\text { Other degenerative diseases of } \\
\text { the nervous system }\end{array}$ \\
\hline J60-J70 & 5 & $1(0.04 \%$ o $)$ & $0(0.00 \%$ o $)$ & $3(0.52 \%$ o $)$ & $1(0.24 \%$ o $)$ & 0.026 & $\begin{array}{l}\text { Lung diseases due to external } \\
\text { agents }\end{array}$ \\
\hline K55-K64 & 5 & $1(0.04 \%$ o $)$ & $0(0.00 \%)$ & $3(0.52 \%)$ & $1(0.24 \%$ o $)$ & 0.026 & Other diseases of intestines \\
\hline
\end{tabular}

Values for the MC groups are count of codes for primary and secondary causes of death and incidence within MC group in per-mille. $P$ values are results from $\chi^{2}$ tests for trend in proportions.

associated with all-cause mortality $(P<0.001, P<0.05$, and $P<0.05$, respectively). The most vulnerable participants were found in the groups with poor function and adverse MC [HRs 5.7 (3.6-9.1), $P<0.001$, for low hand grip strength and adverse $M C$ vs. high hand grip strength without adverse MC; 5.4 (3.3-8.7), $P<0.001$ for slow walking pace and $a d-$ verse $M C$ vs. average/brisk walking pace without adverse $M C ; 4.0$ (2.0-8.1), $P<0.001$ for $>1$ fall last year and adverse $M C$ vs. no falls last year without adverse $M C$.

\section{Discussion}

In this work, we present results from the large UK Biobank imaging study illustrating the impact of muscle composition on all-cause mortality. The main findings include that (i) adverse $M C$ was significantly, and independently, associated with all-cause mortality with a magnitude of HR comparable with that of previous cancer diagnosis or smoking, (ii) adverse $M C$ was highly associated with all-cause mortality, both within those with good function and within those with poor function, and (iii) the combination of poor function with adverse $M C$ identified the most vulnerable participants.

Adverse MC combines measurements of muscle fat infiltration and fat-free muscle volume (in a sex-invariant and sex, weight, and height invariant muscle volume z-score).
Although there is a growing body of evidence supporting the importance of myosteatosis, ${ }^{7,11-14,20-24}$ the literature on associations of muscle quantity (usually muscle mass) with adverse outcomes is inconsistent. ${ }^{4,13,15,17-19,30}$ Ambiguous results on the predictive performance of muscle mass could be attributed to studies using different techniques (e.g. BIA, DXA, $\mathrm{D}_{3} \mathrm{Cr}, \mathrm{CT}$, or MRI), and different methods for quantification based on acquired images (e.g. segmentation and calculation), and for body size adjustment. Recent investigations on different ways of normalizing muscle quantity for body size has, for example, shown that division by height ${ }^{2}$ and weight results in strong correlations with BMI but with inverse directions of association. ${ }^{13}$ Further, a NHANES III study showed that these two ways of calculating skeletal muscle in$\operatorname{dex}(\mathrm{SMI})$, measured by BIA (i.e. SMI=muscle mass/height ${ }^{2}$ and $\mathrm{SMI}=$ muscle mass/weight), for sarcopenia detection also resulted in inversely directional associations with NAFLD. ${ }^{31}$ Our study showed that muscle volume z-score was significantly associated with mortality independent of both myosteatosis and hand grip strength, and in contrast to hand grip strength, remained significant when applying full adjustment.

In this study, the thigh muscles were used to assess muscle volume and fat infiltration. Studies on sarcopenia and wasting disorders have utilized different muscles or groups to assess muscle health (e.g. total or appendicular lean mass, 


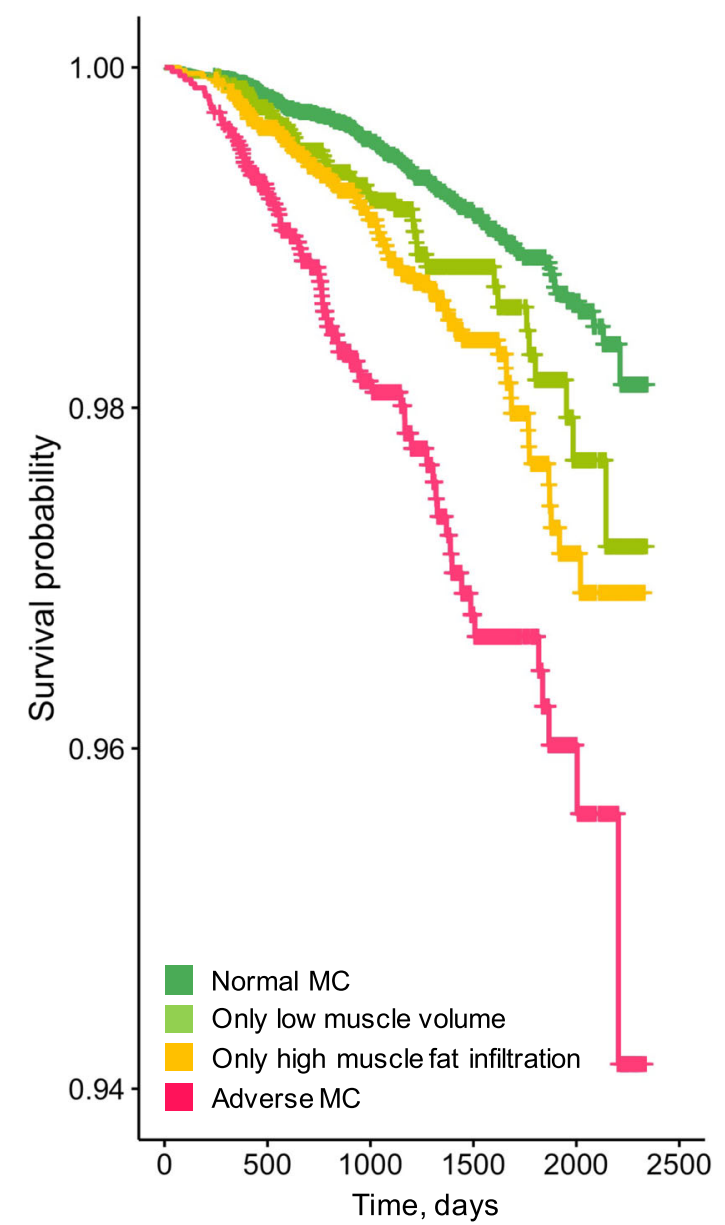

Figure 2 Kaplan-Meier survival curves for all-cause mortality across muscle composition (MC) groups.

total lumbar muscle cross-sectional area, or single muscles like psoas major) ${ }^{3,32}$ but no studies, to our knowledge, have been performed that compare the predictive performance of different muscle groups for specific diseases and outcomes. From the existing MRI data in UK Biobank, several different muscles can be assessed and could provide a greater understanding of a patient's overall muscle health by capturing different aspects of a disease process, everyday functional fitness, and the resulting quality of life.

The breakdown of primary and secondary causes of death into ICD-10 blocks indicated that adverse $M C$ seems to be associated with increased mortality risk across many different disease areas. Although interestingly for some ICD-10 blocks, only high MFI was associated with similar (or higher) mortality rates as adverse MC. It has been shown that low muscle mass and myosteatosis are weakly correlated with each other and they seem to represent two different biological processes involved in muscle wasting. ${ }^{13,14,22,24}$ Results from this study on the non-significant interaction between muscle volume and MFI with all-cause mortality further strengthens this hypothesis. That the mechanisms causing differences in muscle composition may differ should be considered when developing new treatment and prevention strategies.

Although the associations between mortality and hand grip strength (both continuously and binary represented) were attenuated when models were adjusted for previous disease, the associations were borderline significant and the results indicate that $\mathrm{MFI}$, muscle volume $\mathrm{z}$-score, and hand grip strength could effectively aid in the identification of vulnerable individuals independent of each other. The predictive promise of such combination was further strengthened by the analysis combining adverse $M C$ with measures of functional performance and frailty (low hand grip strength, slow walking pace, and $>1$ fall last year) where the most vulnerable individuals were those with poor function and adverse MC. However, even though participants had good functional performance (high hand grip strength, average/brisk walking pace, and 0-1 falls last year), adverse $M C$ was still associated with increased mortality with HRs as high has 2.5-2.9 compared with those without adverse MC. This indicates that knowing a patient's muscle composition could be valuable even though they do not yet express poor function or frailty. However, detection of adverse $M C$ requires tomographic imaging (MRI or CT), which is not readily available in many clinical workflows today. In certain disease populations, such as end-stage liver disease or heart failure, MRI is commonly used and thus, adverse $M C$ could more easily be assessed and potentially aid in identifying high-risk patients. It has been shown that low muscle mass and myosteatosis is associated with higher mortality in patients with cirrhosis and a patient's muscle composition may affect the outcome of disease interventions like liver transplantation. ${ }^{11,33}$ In workflows where tomographic imaging is less available and resources to intervene are scarce, other tests that have shown predictive performance for sarcopenia related outcomes, like hand grip strength, walking pace or $\mathrm{D}_{3} \mathrm{Cr}$ dilution, could be used to identify who may benefit most from a muscle composition assessment. One benefit of volumetric MRI-based muscle composition assessment is the high precision allowing close tracking of muscle wasting and disease progression ${ }^{28}$ - something that is not as important for diagnostic purposes but imperative during development and evaluation of new treatments. The main challenge is the access to, and cost of, MRI.

Reaching standardized and accurate ways to measure muscle quantity and quality is highly important for future studies or clinical applications of techniques to assess sarcopenia or muscle wasting. A challenge with DXA is that it does not measure muscle mass directly. For example, the measurement of lean muscle mass by DXA includes water and fibrotic tissue and thus confounds the muscle assessment depending on the patient's status. ${ }^{34}$ Muscle mass as assessed by $D_{3} \mathrm{Cr}$ dilution has shown strong relation with physical performance, incidence of falls, mobility limitations, and mortality but does 


\begin{tabular}{|c|c|c|c|c|c|}
\hline Variable & & $\mathbf{N}$ & Hazard ratio & & $\mathbf{p}$ \\
\hline \multirow[t]{4}{*}{ Muscle composition (MC) } & normal mc & 22905 & 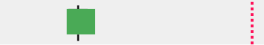 & Reference & \\
\hline & only low muscle volume & 5439 & $\longmapsto \quad 1$ & $1.11(0.78,1.59)$ & 0.56 \\
\hline & only high muscle fat infiltration & 5477 & -1 & $1.49(1.08,2.07)$ & 0.02 \\
\hline & adverse mc & 3860 & - & $1.96(1.42,2.71)$ & $<0.001$ \\
\hline \multirow[t]{2}{*}{ Low hand grip strength } & no & 34945 & 中 & Reference & \\
\hline & yes & 2736 & $\mathbf{a}$ & $1.34(0.96,1.88)$ & 0.09 \\
\hline \multirow[t]{2}{*}{ Sex } & female & 19520 & $\square$ & Reference & \\
\hline & male & 18161 & 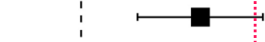 & $1.60(1.25,2.05)$ & $<0.001$ \\
\hline Age, years & & 37681 & $\mathbf{u}$ & $1.09(1.07,1.11)$ & $<0.001$ \\
\hline $\mathrm{BMI}, \mathrm{kg} / \mathrm{m}^{2}$ & & 37681 & 1 & $0.98(0.95,1.01)$ & 0.28 \\
\hline \multirow[t]{2}{*}{ Cancer } & no & 33720 & 口 & Reference & \\
\hline & yes & 3961 & - & $1.93(1.47,2.53)$ & $<0.001$ \\
\hline \multirow[t]{2}{*}{ Type 2 diabetes } & no & 35779 & 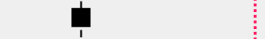 & Reference & \\
\hline & yes & 1902 & - & $1.58(1.09,2.29)$ & 0.02 \\
\hline \multirow[t]{2}{*}{ Coronary heart disease } & no & 35759 & 1 & Reference & \\
\hline & yes & 1922 & 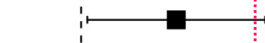 & $1.46(1.03,2.07)$ & 0.04 \\
\hline \multirow[t]{3}{*}{ Smoking status } & no & 23601 & Q & Reference & \\
\hline & previous & 12760 & -1 & $1.10(0.86,1.40)$ & 0.45 \\
\hline & current & 1320 & 1 & $1.71(1.02,2.84)$ & 0.04 \\
\hline Alcohol consumption, g/day & & 37681 & $\varphi$ & $1.00(1.00,1.00)$ & 0.81 \\
\hline \multirow[t]{3}{*}{ Physical activity } & moderate & 16535 & $\dot{1}$ & Reference & \\
\hline & low & 7409 & 一 & $1.37(1.03,1.82)$ & 0.03 \\
\hline & high & 13737 & - & $1.21(0.93,1.58)$ & 0.15 \\
\hline Townsend deprivation index & & 37681 & - & $1.02(0.98,1.06)$ & 0.35 \\
\hline
\end{tabular}

Figure 3 Cox proportional-hazard ratios of all-cause mortality for muscle composition (MC) phenotypes (green, light green, yellow, and pink) adjusted for low hand grip strength, sex, age, body mass index BMI, previous diagnosis of disease, lifestyle- and socioeconomic factors.

not measure myosteatosis. ${ }^{17,18}$ Measurements of muscle mass and myosteatosis is achievable with $\mathrm{CT}$. However, $\mathrm{CT}$ is limited by the substantial ionizing radiation, which hinders its applicability for wider research purposes or for the sole purpose of body/muscle composition assessment. In addition, factors like tube voltage, equipment calibration, slice thickness, use of contrast agents and phases can affect the skeletal muscle radiodensity and the resulting measurement of the amount of muscular mass and fat. ${ }^{35-37}$ Body and muscle composition is not commonly assessed from clinically obtained MRI images, but opportunistic assessment of body/muscle composition based on MRI would face similar challenges as $\mathrm{CT}$ with regard to standardization. The MRI protocol and process used to measure thigh muscle volume and fat infiltration in this study has shown high repeatability and reproducibility across common MR scanners (GE, Philips, and Siemens) and different magnetic field strengths (1.5 and $3 \mathrm{~T}) .^{28}$

The strengths of this work include the high number of participants included, the high-quality muscle biomarkers, and the access to proper measurements of hand grip strengths. The main limitations were the relatively short observation time and few deaths in relation to cohort size. In addition, although modelling included adjustment with previous diseases (cancer, type 2 diabetes, and coronary heart disease) as well as other factors that have been shown to associate with increased mortality risk, time of onset was not available for all conditions (adverse MC and previous diseases) and was not accounted for in the analysis. It is not possible from these study results to conclude whether diseases cause worsening of the muscle composition, or if adverse $M C$ contributes to the aggravation of diseases. In the latter case, assessing muscle composition could potentially help identify patients that have a poor prognosis and guide treatment strategies. Lastly, there is evidence of UK Biobank exhibiting a 'healthy volunteer' selection bias. ${ }^{38}$ However, conclusions on associations between exposures and health outcomes are generalizable to the wider population because of the large sample size and heterogeneity of exposure measures. ${ }^{38}$ 


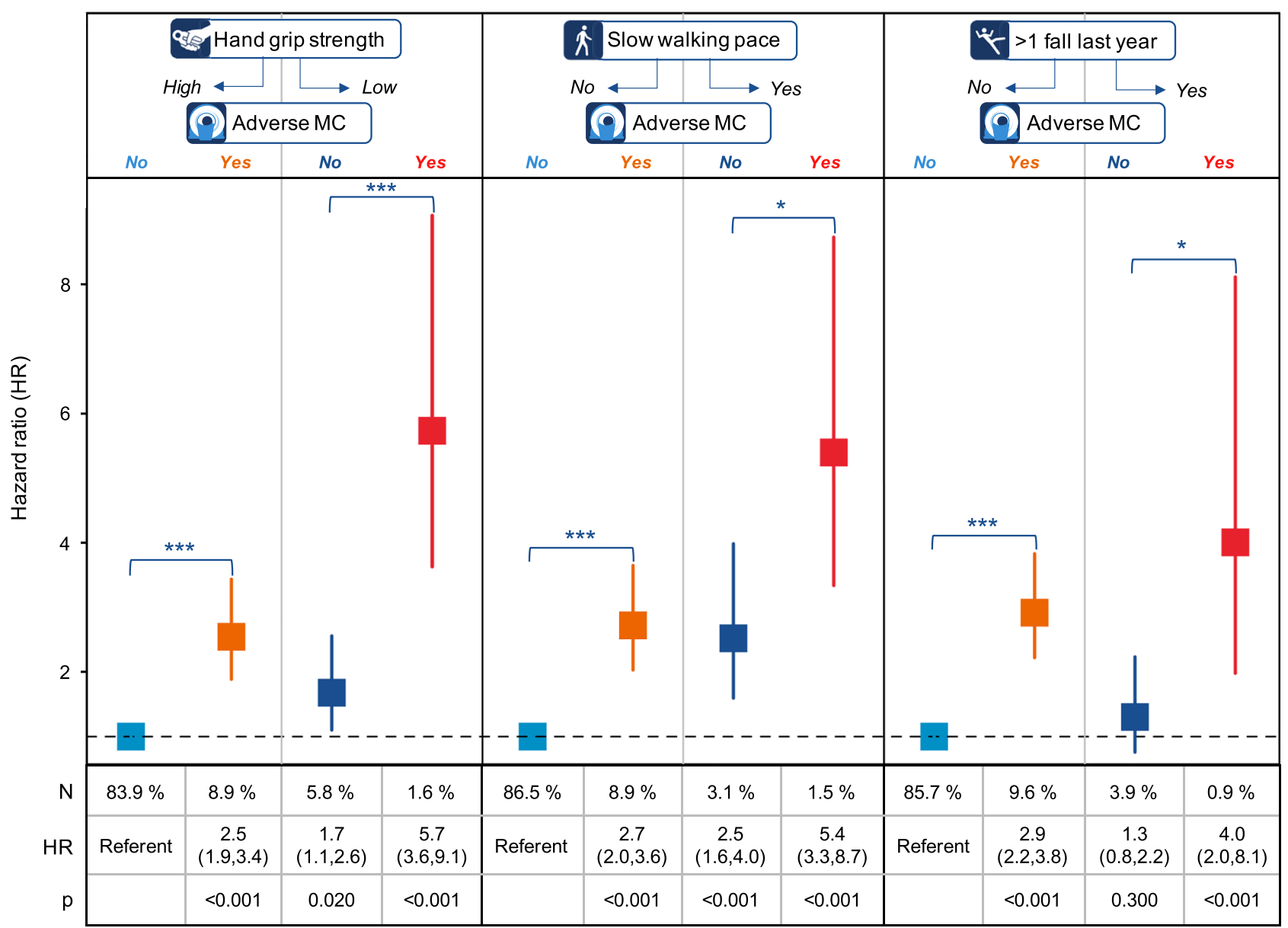

Figure 4 Cox proportional-hazard ratios (unadjusted) of all cause-mortality for the combined assessment of functional performance (high or low hand grip strength, slow or average/brisk).

Future analysis should investigate aspects of causality, specific causes of death and disease specific cut-points to identify high-risk patients based on muscle composition assessment. Such investigations would be most beneficial to perform for disease populations where tomographic imaging is more commonly used today. It should be noted that the cut-points published in this paper are based on a general population and may be too inclusive if applied to specific patient populations that more commonly suffer from sarcopenia related issues. In addition, UK Biobank is a relatively young cohort, and age might influence what is an optimal threshold for identifying vulnerable patients, especially for myosteatosis. ${ }^{39}$

\section{Conclusions}

Adverse MC was a strong, and independent, predictor of all-cause mortality. The combination of adverse MC and poor function identified the most vulnerable participants.
Including cut-offs for myosteatosis to enable detection of adverse $M C$ could strengthen sarcopenia guidelines.

\section{Conflict of interest}

All authors have completed the ICMJE uniform disclosure form online (www.icmje.org/coi_disclosure.pdf; available on request from the corresponding author). J. L., M. F. F., M. P., and O. D. L. reports other from Pfizer Inc., during the conduct of the study; other from AMRA Medical, outside the submitted work. In addition, J. L. and O. D. L. have a patent METHOD OF EVALUATING A MUSCLE RELATED CONDITION pending to PCT/EP2020/053068. A. J. S. reports grants from Intercept, during the conduct of the study; other from Sanyal Bio, Genfit, Indalo, Tiziana, Durect, Exhalenz, Galmed, second genome, Cymabay, Prosciento, Labcorp, Medimmune, Astra Zeneca, Albireo; grants from Merck, Bristol Myers, Boehringer Ingelhiem, Immuron, Malinkrodt, Cumberland, Sequana; grants and personal fees from Novartis, Gilead, 
Conatus, Echosens; personal fees from Pfizer, Lilly, Novo Nordisk, Sanofi, Tern, Hemoshear, Glympse, Birdrock, Blade, Teva, Artham, Salix, NASH pharmaceuticals, outside the submitted work.

\section{Funding}

Pfizer Inc.; European Union's Horizon 2020 Research and Innovation Programme (CoMorMent project; Grant \#847776).

\section{Ethics statement}

The authors of this manuscript certify that they comply with the ethical guidelines for authorship and publishing in the Journal of Cachexia, Sarcopenia and Muscle. ${ }^{40}$

\section{Online supplementary material}

Additional supporting information may be found online in the Supporting Information section at the end of the article.

Table S1. Cox proportional-hazard ratios of all-cause mortality for muscle composition variables (M0).

Table S2. Cox proportional-hazard ratios of all-cause mortality for muscle composition variables adjusted for hand grip strength (M1).

Table S3. Cox proportional-hazard ratios of all-cause mortality for muscle composition variables adjusted for hand grip strength, sex, age and BMI (M2).

Table S4. Cox proportional-hazard ratios of all-cause mortality for muscle composition variables adjusted for hand grip strength, sex, age, BMI and previous disease (type 2 diabetes, coronary heart disease and cancer) (M3).

Table S5. Cox proportional-hazard ratios of all-cause mortality for muscle composition variables adjusted for hand grip strength, sex, age, BMI, previous disease (type 2 diabetes, coronary heart disease and cancer), lifestyle- and socioeconomic factors (M4).

Table S6. Cox proportional-hazard ratios of all-cause mortality for muscle composition (MC) phenotypes (M0).

Table S7. Cox proportional-hazard ratios of all-cause mortality for muscle composition (MC) phenoptypes adjusted for low hand grip strength (M1).

Table S8. Cox proportional-hazard ratios of all-cause mortality for muscle composition (MC) phenotypes adjusted for hand grip strength, sex, age and BMI (M2).

Table S9. Cox proportional-hazard ratios of all-cause mortality for muscle composition (MC) phenotypes adjusted for hand grip strength, sex, age, BMI and previous disease (type 2 diabetes, coronary heart disease and cancer) (M3).

Table S10. Cox proportional-hazard ratios of all-cause mortality for muscle composition (MC) phenotypes adjusted for hand grip strength, sex, age, BMI, previous disease (type 2 diabetes, coronary heart disease and cancer), lifestyle- and socioeconomic factors (M4).

Figure S1. Scatter plot of muscle volume z-score and muscle fat infiltration with corresponding sex-specific density plots (blue $=$ females, red $=$ males) showing the distributions of each variable on top and to the left respectively. Dashed lines indicate the cut-offs for identification of Adverse muscle composition.

Figure S2. The most common specific primary and secondary causes of death divided into ICD-10 code blocks and number of codes (per mille) across muscle composition (MC) phenotypes.

\section{References}

1. Bone AE, Hepgul N, Kon S, Maddocks M. Sarcopenia and frailty in chronic respiratory disease. Chron Respir Dis 2017;14:85-99.

2. Bahat G, Ilhan B. Sarcopenia and the cardiometabolic syndrome: a narrative review. Eur Geriatr Med 2016;7:220-223.

3. Cruz-Jentoft AJ, Bahat G, Bauer J, Boirie Y, Bruyère $O$, Cederholm $T$, et al. Sarcopenia: revised European consensus on definition and diagnosis. Age Ageing 2019;48:16-31.

4. Cawthon PM, Manini T, Patel SM, Newman A, Travison T, Kiel DP, et al. Putative cut-points in sarcopenia components and incident adverse health outcomes: an SDOC analysis. J Am Geriatr Soc 2020;68 1429-1437.

5. Volpi E, Nazemi R, Fujita S. Muscle tissue changes with aging. Curr Opin Clin Nutr Metab Care 2004;7:405-410.

6. Mesinovic J, Zengin A, De Courten B Ebeling PR, Scott D. Sarcopenia and type 2 diabetes mellitus: a bidirectional relationship. Diab Metab Syndr Obes 2019;12 1057-1072.

7. Chakravarthy MV, Siddiqui MS, Forsgren MF, Sanyal AJ. Harnessing muscle-liver crosstalk to treat nonalcoholic steatohepatitis. Front Endocrinol
(Lausanne) 2020;11592373, https://doi org/10.3389/fendo.2020.592373

8. Fearon K, Strasser F, Anker SD, Bosaeus I, Bruera $E$, Fainsinger $R L$, et al. Definition and classification of cancer cachexia: an international consensus. Lancet Oncol 2011;12:489-495.

9. Dasarthy S. Etiology and management of muscle wasting in chronic liver disease. Curr Opin Gastroentrol 2016;32: 159-165.

10. Mak RH, Ikizer AT, Kovesdy CP, Raj DS, Stenvinkel P, Kalantar-Zadeh K. Wasting in 
chronic kidney disease. I Cachexia Sarcopenia Muscle 2011;2:9-25.

11. Montano-Loza AJ, Angulo P, Meza-Junco J, Prado CMM, Sawyer MB, Beaumont C, et al. Sarcopenic obesity and myosteatosis are associated with higher mortality in patients with cirrhosis. J Cachexia Sarcopenia Muscle 2016;7:126-135.

12. Nachit M, Leclercq IA. Emerging awareness on the importance of skeletal muscle in liver diseases: time to dig deeper into mechanisms! Clin Sci (Lond) 2019;133: 465-481.

13. Linge J, Heymsfield SB, Dahlqvist LO. On the definition of sarcopenia in the presence of aging and obesity-initial results from UK Biobank. J Gerontol A Biol Med Sci 2020;75:1309-1316.

14. Linge J, Ekstedt M, Dahlqvist LO. Adverse muscle composition is linked to poor functional performance and metabolic comorbidities in NAFLD. JHEP Rep 2020;3:100197, https://doi.org/10.1016/j. jhepr.2020.100197

15. Newman AB, Kupelian V, Visser $M$, Simonsick EM, Goodpaster BH, Kritchevesky SB, et al. Strength, but not muscle mass, is associated with mortality in the health, aging and body composition study cohort. J Gerontol A Biol Sci Med Sci 2006;61:72-77.

16. Batsis AJ, Mackenzie TA, Emeny RT, Lopez-Jimenez F, Bartels SJ. Low lean mass with and without obesity, and mortality: results from the 1999-2004 National Health and Nutrition Examination Survey. J Gerontol A Biol Sci Med Sci 2017;72:1445-1451.

17. Cawthon PM, Blackwell T, Cummings SR, Orwoll ES, Duchowny KA, Kado DM, et al. Muscle mass assessed by the D3-creatine dilution method and incident self-reported disability and mortality in a prospective observational study of community-dwelling older men. J Gerontol A Biol Sci Med Sci 2021;76:123-130.

18. Cawthon PM, Orwoll ES, Peters KE, Ensrud KE, Cauley JA, Kado DM, et al. Strong relation between muscle mass determined by D3-creatine dilution, physical performance, and incidence of falls and mobility limitations in a prospective cohort of older men. J Gerontol A Biol Sci Med Sci 2019;74: 844-852.

19. Linge J, Ekstedt M, Dahlqvist LO. Reply to: "Rationale of adding muscle volume to muscle fat infiltration in the definition of an adverse muscle composition is unclear". JHEP Rep 2021;3:100257, https://doi.org/ 10.1016/j.jhepr.2021.100257
20. Correa-de-Araujo R, Addison O, Miljkovic I, Goodpaster BH, Bergman BC, Clark RV, et al. Myosteatosis in the context of skeletal muscle function deficit: an interdisciplinary workshop at the National Institute on Aging. Front Physiol 2020;11:963.

21. Rier HN, Jager A, Sleijfer S, van Rosmalen J, Kock MCJM, Levin MD. Low muscle attenuation is a prognostic factor for survival in metastatic breast cancer patients treated with first line palliative chemotherapy. Breast 2017;31:9-15.

22. Loosen $S H$, Schulze-Hagen $M$, Püngel $T$, Bündgens L, Wirtz T, Kather JN, et al. Skeletal muscle composition predicts outcome in critically ill patients. Crit Care Explor 2020;2:e0171, https://doi.org/10.1097/ CCE. 0000000000000171

23. Kumar A, Moynagh MR, Multinu F, Cliby WA, McGree ME, Weaver AL, et al. Muscle composition measured by $\mathrm{CT}$ scan is a measurable predictor of overall survival in advanced ovarian cancer. Gynecol Oncol 2016;142:311-316.

24. Stretch C, Aubin JM, Mickiewicz B, Leugner D, Al-Manasra T, Tobola E, et al. Sarcopenia and myosteatosis are accompanied by distinct biological profiles in patients with pancreatic and periampullary adenocarcinomas. PloS one 2018;13:e0196235, https://doi.org/10.1371/journal. pone.0196235

25. Linge J, Borga $M$, West J, Tuthill $T$, Miller MR, Dumitriu A, et al. Body composition profiling in the UK Biobank imaging study. Obesity (Silver Spring) 2018;26: 1785-1795.

26. Karlsson A, Rosander J, Romu T, Tallberg J, Grönqvist A, Borga $M$, et al. Automatic and quantitative assessment of regional muscle volume by multi-atlas segmentation using whole-body water-fat MRI. J Magn Reson Imaging 2015;41:1558-1569.

27. West J, Romu T, Thorell $S$, Lindblom $H$, Berin E, Spetz Holm AC, et al. Precision of MRI-based body composition measurements of postmenopausal women. PLOS One 2018;13:e0192495.

28. Borga $M$, Ahlgren A, Romu T, Widholm $P$, Dahlqvist Leinhard O, West J. Reproducibility and repeatability of MRI-based body composition analysis. Magn Reson Med 2020;84:3146-3156.

29. Sudlow C, Gallacher J, Allen N, Beral V, Burton P, Danesh J, et al. UK Biobank: an open access resource for identifying the causes of a wide range of complex diseases of middle and old age. PLoS Med 2015;12: e1001779.

30. Santanasto AJ, Miljkovic I, Cvejkus RC, Gordon $\mathrm{CL}$, Bunker $\mathrm{CH}$, Patrick AL, et al.
Body composition remodeling and incident mobility limitations in African ancestry men. J Gerontol A Biol Sci Med Sci 2019;74:400-405.

31. Peng TC, Wu LW, Chen WL, Liaw FY, Chang YW, Kao TW. Nonalcoholic fatty liver disease and sarcopenia in a Western population (NHANES III): the importance of sarcopenia definition. Clin Nutr 2019;38: 422-428.

32. Gu DH, Kim MY, Seo YS, Kim SG, Lee HA, $\mathrm{Kim} \mathrm{TH}$, et al. Clinical usefulness of psoas muscle thickness for the diagnosis of sarcopenia in patients with liver cirrhosis. Clin Mol Hepatol 2018:24:319-330.

33. Carey EJ, Lai JC, Sonnenday C, Tapper EB, Tandon P, Duarte-Rojo A, et al. A North American expert opinion statement on sarcopenia in liver transplantation. Hepatology 2019;70:1816-1829.

34. Prado CMM, Heymsfield SB. Lean tissue imaging: a new era for nutritional assessment and intervention. JPEN J Parenter Enteral Nutr 2014;38 (8):940-953.

35. Poltronieri TS, de Paula NS, Chaves G. Assessing skeletal muscle radiodensity by computed tomography: An integrative review of the applied methodologies. Clin Physiol Funct Imaging 2020;40:207-223.

36. Fuchs G, Chretien YR, Mario J, Do $S$, Eikermann $M$, Liu B, et al. Quantifying the effect of slice thickness, intravenous contrast and tube current on muscle segmentation: implications for body composition analysis. Eur Radiol 2018;28:2455-2463.

37. van der Werf A, Dekker IM, Meijerink MR, Wierdsma NJ, de van der Schueren MAE, Langius JAE. Skeletal muscle analyses: agreement between non-contrast and contrast CT scan measurements of skeletal muscle area and mean muscle attenuation. Clin Physiol Funct Imaging 2018;38: 366-372.

38. Fry A, Littlejohns TJ, Sudlow C, Doherty N, Adamska L, Sprosen T, et al. Comparison of sociodemographic and health-related characteristics of UK Biobank participants with the general population. Am J Epidemiol 2017;186:1026-1034.

39. Marcus RL, Addison O, Kidde JP, Dibble LE, Lastayo PC. Skeletal muscle fat infiltration: impact of age, inactivity, and exercise. $J$ Nutr Health Aging 2010;14 (5):362-366.

40. von Haehling S, Morley JE, Coats AJS, Anker SD. Ethical guidelines for publishing in the Journal of Cachexia, Sarcopenia and Muscle: update 2019. J Cachexia Sarcopenia Muscle 2019;10:1143-1145. 